\title{
Bluetooth Ad hoc Network
}

\author{
Shinde P.R ${ }^{1}$, Sutar A.J \\ Computer Science and Engineering, AGCE Satara, Satara, India ${ }^{1,2}$
}

\begin{abstract}
In the Disaster (e.g. Natural disaster like earthquake, cyclone etc) situation the communication medium goes down because of high congestion the network is unavailable. In that situation it is important to exchange information .In the disaster situation, due to the high congestion the communication infrastructure is partially or totally goes down or unavailable. However, during such critical situations, there is an acute need for information exchange for both rescue organizations and victims (person in disaster). Temporary communication solutions are important until the infrastructure is restored. In this paper, we start by reviewing communication solutions - By using the simple technology which is used everyone - able to uphold communications during disasters when the communication infrastructure is destroyed. We present how these solutions can be applied and summarize the advantages and disadvantages of each unique approach. In a second part, we present One Android application for establishing the communication. The application is based on the simple technology named as Bluetooth and using that communicates the Rescue team and victims. In that we create the one disaster mode. In the disaster mode, the one who present in the disaster area those takes admin role and searches nearest Bluetooth devices and connect and create one small AD-HOC network. In other side the victim also use the application and turn on the disaster mode and send the rescue message to the rescue team (rescue team is the admin or nearest Bluetooth device). Simply, we demonstrate how these useful technologies such as Bluetooth can be of great value right after a disaster by enabling the self-organization of victims and a better coordination with first rescue team.
\end{abstract}

Keywords: Bluetooth, Pico net, Disaster.

\section{INTRODUCTION}

In cellular network such as wired telecommunication system the services are modern and flexible. In natural disaster i.e in terrorist attacks, floods, in disaster situation i.e earthquakes. when our cellular network goes down because of disturbance in cellular network at that time communication with each other totally get down. In this situation peoples can't communicate with each other. In situation like rout the peoples don't reach to their family or friends.

In this paper, for solution we are developing an Android application. In Android application there is disaster mode. When we turn on our disaster mode. It will search nearest helpful person. In our android application we create the two application one is the admin and another is the victims (person who is in the disaster) The admin application helps the victims like the admin create the one small AD-HOC network and connect the nearest Bluetooth devices.

The admin is the who which are the nearest Bluetooth device in disaster area they take a roll of admin. And victim are the whose person which are in the disaster situation. In second part the victims also use the application and on the disaster mode when the disaster mode is on then it searches the nearest Bluetooth devices (rescue team). And send the rescue message to the rescue team. At that time question is arise the what is the roll of admin

The admin is responsible to create the small network and connect the multiple hopes in that and using that hopes we reaches the victim or rescue person. After that victims application searches the nearest rescue hope and send the one formatted message to the rescue team.

\section{SURVEY OF TECHNOLOGY}

We have taken the survey of technology which provides the communication services in disaster relief situation. We focus on the how our prototype will work with this technology. Survey technology like Cellular Network, Wireless networks.

\subsection{Classification}

Cellular Network

Cellular Networks operates on wide range. It provides communication services at the coverage area as per service provider decided. It works on cell structure like pentagons, hexagons etc.

Cellular networks may requires a infrastructure and high maintenance. Cellular network requires base stations, antennas, MSTN (Mobile Switching Telephone Network), PSTN (Public Switched Telephone Network) for get communication with user who are at within range coverage area.

\section{A. Architecture of Bluetooth}

Below diagram represents architecture of wireless Bluetooth Technology. In this technology simply use a Pico net architecture to broadcast Ana log signals as master node to connect devices which works as slaves for sharing of data or exchanging information. 
IARJSET

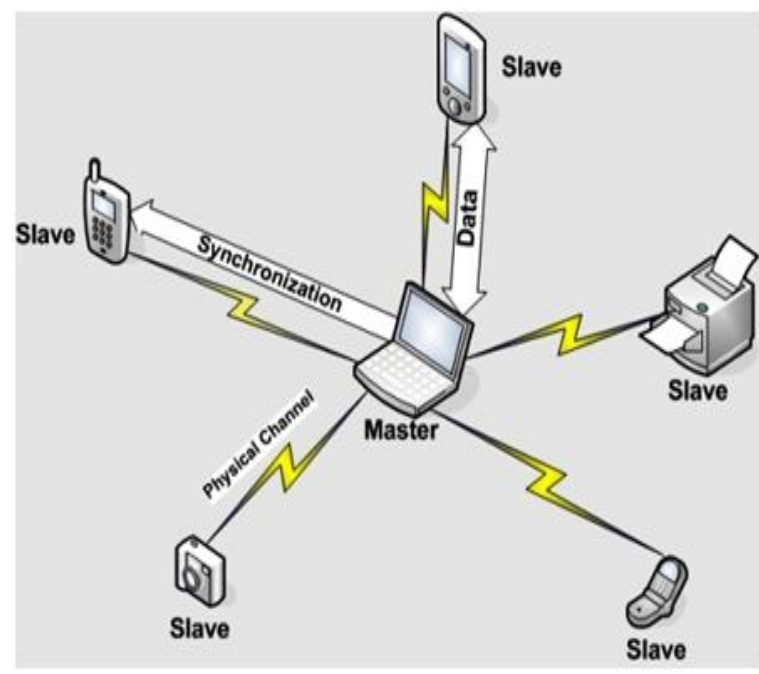

Fig. Architecture of Bluetooth Technology

\section{B. Bluetooth technology}

Bluetooth can be used for connecting media devices which are in short rang. The rang between devices are communicate with other is in between 10 to $100 \mathrm{~m}$.Every device has a transceiver chip through that it receive or transmit unlicensed IMS frequency band of $2.4 \mathrm{GHz}$ which is in globally available. With this frequency band the devices are communicate each other. Bluetooth devices are connected one to one or one to multiple. In these devices one node is act as master $\mathrm{n}$ another is act as slave. The master node transfer data to another slave which is connected to them. Slave who are connected to the master are any number but only 7 slaves are active at that time. The features of Bluetooth include robustness, require less power, less cost. There are three version of Bluetooth are available in market are: 1.0, 1.0b And 1.1

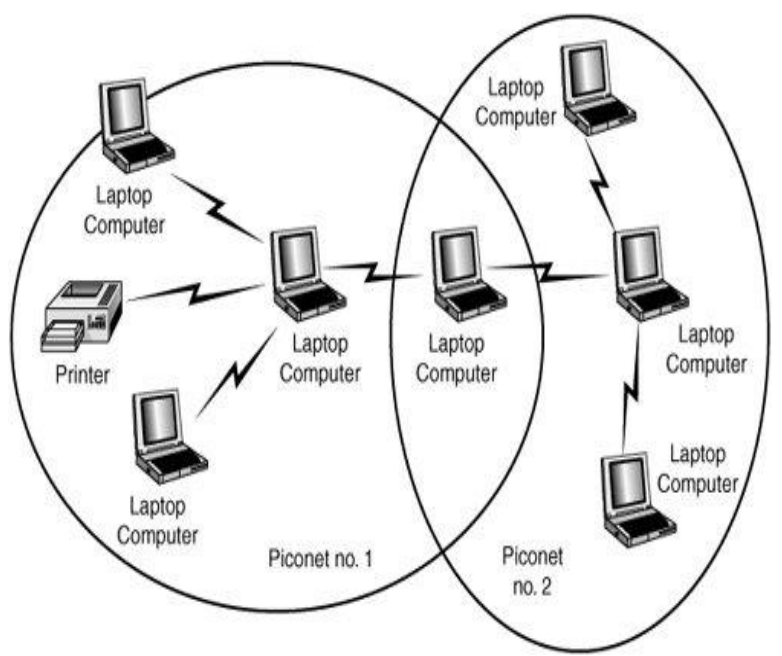

Fig. Pico net \& Scatter net Architecture

Master Bluetooth device can communicate with maximum seven device in each Pico net.(ad-hoc network) above seven device it can't get communicate. In Bluetooth technology when communication get possible at that time in one Pico net, there will be one master Bluetooth device an another are slave. In one Pico net structure only seven Bluetooth device get communicate. Bluetooth technology is connection-oriented technology which requires at least two device for communication. Bluetooth uses LMP, L2CAP, SDP Protocols. LMP protocol is used to setup and control on the radio frequency between two devices.

When communication get possible at that time it uses the MAC address of nearest Bluetooth. It totally works on MAC address as well as on radio frequency.

When Bluetooth search nearest device after device discovery enable. It look for device name, class, and unique MAC address.

\section{Basic Operation}

When a Bluetooth device is powered on, it may try to operate as one of the slave devices of an already running master device. It then starts listening for a master's inquiry for new devices and responds to it. The inquiry phase lets the master know the address of the slave; this phase is not necessary for very simple paired devices that are granted to know each other's address. Once a master knows the addressof a slave, it may open a connection toward it, provided the slave is listening for paging requests. If this is the case, the slave responds to the master's page request and the two devices synchronize over the frequency hopping sequence, which is unique to each piconet and decided by the master. Bluetooth predefines several types of connection, each with a different combination of available bandwidth, error protection, and quality of service. Once a connection is established, the devices can optionally authenticate each other and then communicate. Devices not engaged in transmissions can enter one of several power and bandwidth-saving modes or tear down the connection. Master and slave can switch roles, which may be necessary when a device wants toparticipate in more than one piconet.

\section{III.METHODOLOGY}

If the disaster can happen it gets effect on the communication infrastructure. When there is no any communication infrastructure the victim can't exchange the information with other person. For communication or information exchange we need a temporary wireless media at that time of the disaster. A solution to the disaster situation we are working on the project, which can help to connecting with person who in disaster.

There are various wireless media are present for communication in market. One of them we can use in our project which is a simple "Bluetooth". As we see, there are various technologies in the market for wireless network. But the reason behind for using Bluetooth technology is because it can uses less power for its performance.

\section{A. DFD level 0}

Below data flow diagram represents, a single user activates his disaster mode and send message to a rescue team. Again a rescue sends Acknowledgment to a user. 
IARJSET

Ack

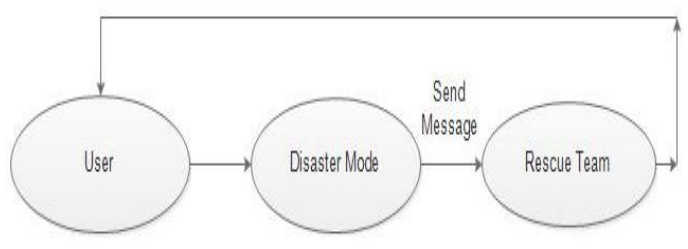

Fig.DFD level 0

B. DFD level 1

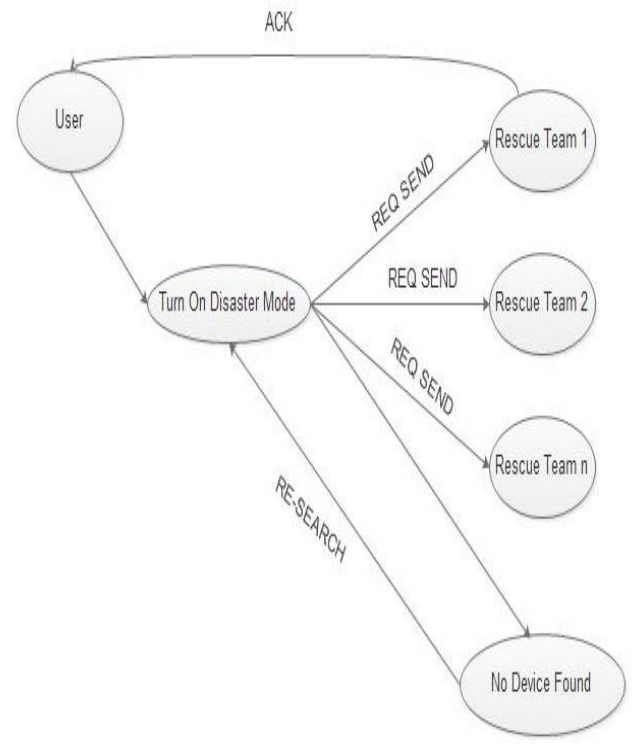

Fig. DFD level 1

Above Data flow diagram is level of one which illustrate that user make his disaster mode on and send to number of rescue teams. If rescue team receive that request then it will send a acknowledgment to user again. Else it will get a message like no device found and again process will go into searching mode.

\section{Sequence Diagram}

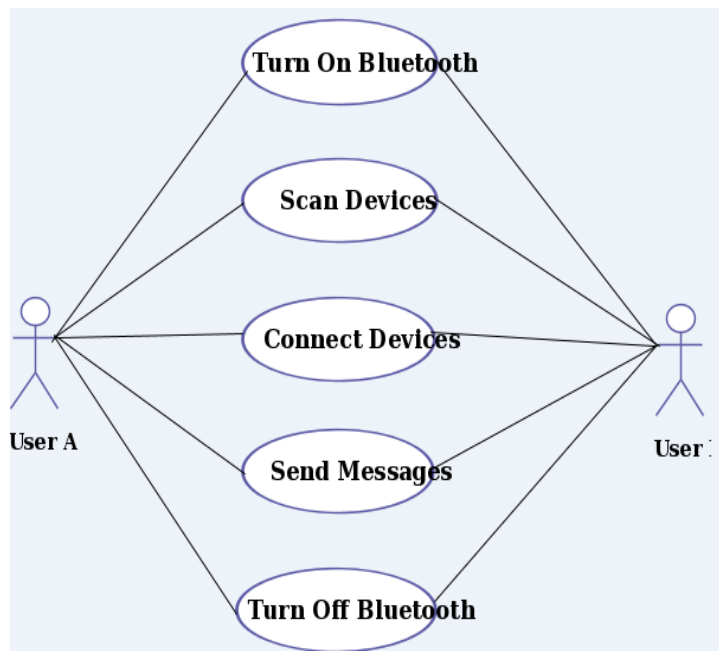

Fig. Sequence Diagram
Above sequence diagram describe a connection between user $A$ and user B through number of different phases like turn on Bluetooth, scan device, connect devices, send message and turn off Bluetooth. After passing these phases connection will be established cleanly and in a proper way.

\section{IV.CONCLUSION}

When disaster situation will be occurs, there is no any communication infrastructure is available to exchanging a information. So, this paper will help to give solution to communicate people who is in disaster. This paper help to implement an ad-hoc network using Bluetooth technology. Bluetooth technology is one of the wireless technology which helps to exchanging information or sharing data between two devices by using radio waves which helps in a small area. Bluetooth have one functionality that is it have ales power consumption. By using this functionality we use this technology in our android application. By using this application user will easily communicate with rescue team in a crises situation.

\section{REFERENCES}

1. INTRO TO ad hoc networks pdf http://www.cs.jhu.edu/ cs647/intro_adhoc.pdf

2. Bluetooth and wireless protocols.pdf http://fly.isti.cnr.it/pub/papers/pdf/WiFiBluetoothIEEEWCm05.pdf

3. I. Akyildiz, X. Wang, and W. Wang, "Wireless mesh networks: A survey," Computer Networks, vol. 47, no. 4, March 2005.

4. Bluetooth Wikipedia https://www.google.com/bluetooth

\section{BIOGRAPHIES}

Shinde P.R Computer Science and Engineering, AGCE Satara, Satara, India

Sutar A.J Computer Science and Engineering, AGCE Satara, Satara, India 